\title{
Three forms of localized solutions of the quintic complex Ginzburg-Landau equation
}

\author{
V.V. Afanasjev and N. Akhmediev \\ Optical Sciences Centre, The Australian National University, Canberra 0200, Australian Capital Territory, Australia \\ J.M. Soto-Crespo \\ Instituto de Óptica, Consejo Superior de Investigaciones Científicas, Serrano 121, 28006 Madrid, Spain
}

(Received 5 September 1995)

\begin{abstract}
We report numerical observation of two new forms of stable localized solutions of the quintic complex Ginzburg-Landau equation. The first form is a stationary zero-velocity solution, which consists of two motionless fronts and a source between them. We call this structure the "composite" pulse. We show that in some range of parameters, a composite pulse can coexist with a plain pulse solution. At the boundary of their region of existence in the parameter space, composite pulses exhibit a complicated behavior, which includes periodical dynamics and transition into another new form of localized solutions, namely, uniformly translating, or moving pulses. A careful study shows that the moving pulses have an even wider range of existence than the composite pulses. The interactions between different combinations of moving and stationary pulses are also studied. A qualitative explanation of the observed structures is proposed.
\end{abstract}

PACS number(s): 42.25.Bs, 05.70.Ln

\section{INTRODUCTION}

The quintic complex Ginzburg-Landau (CGL) equation has a rich variety of solutions, including localized stationary and uniformly translating structures, periodic and chaotic solutions. In this paper we concentrate on localized solutions, as they are most important for many applications. It is known that the dynamics of localized coherent structures is determined mainly by the competition between pulses and fronts $[1,2]$. If fronts have positive velocity (i.e., if the structure, composed of two fronts, expands), then fronts dominate over pulses. In turn, pulses dominate in the region of negative front velocity.

It was thought that only one stationary stable pulse solution of the quintic CGL equation can exist for a given set of parameters. This follows from the expansions near the conservative limit (nonlinear Schrödinger equation) [3] and purely dissipative limit (real Ginzburg-Landau equation) [4]. Recent analytical studies also supported this hypothesis (see $[5,6]$, and references therein). Indeed, although the variety of the exact solutions of the quintic GL equation has been found [6] (which includes fixed-amplitude pulses, arbitrary-amplitude pulses, algebraic pulses, flat-top pulses), all these solutions can be considered as partial or limiting cases of the general solution. Only one stationary pulse solution has been found in numerical simulations $[7,3,8]$ (we call solution of this type plain pulse).

At the same time, analytic solutions of the quintic GL equation exist only in the codimension-one subspace of the parameter space and these solutions are mostly unstable. Perturbation analysis cannot be applied if the dissipative and conservative effects are of the same order. And numerical simulations cannot cover all range of parameters and an infinite variety of initial conditions.

In this paper we show that there is a certain region in the parameter space where, besides the plain pulse solution, another stable solution, which we call composite pulse (CP), can exist. This region of parameters is smaller than the region of existence of the plain pulses, but this region of parameters is continuous and it has the same dimensionality as for the plain pulses.

Another interesting phenomenon reported in this paper is the existence of uniformly translating nonsymmetric pulse solution, which we call moving pulse (MP). It is usually supposed that only motionless pulse solutions can exist in the quintic CGL equation, if the coefficient in the front of the spectral filtering term is nonzero. This also follows from the perturbation expansion [9] and numerical simulations. However, only symmetric (and motionless) initial conditions have been traditionally used in simulations, so the MP have been missed out. Moreover, moving pulses coexist with plain pulses and composite pulses, so all these three types of solutions can appear simultaneously.

The rest of this paper is organized as follows. In Sec. II we introduce the basic equations and discuss briefly the known approaches to find its solution. In Sec. III we propose the reduction of the CGL equation to a system of three first-order ordinary differential equations (ODE's) and look into its properties. Section IV is devoted to a study of the composite pulses. Rather unexpected uniformly translating asymmetric pulses are discussed in Sec. V. Finally, Sec. VI concludes the paper.

\section{BASIC EQUATION}

We write the quintic complex Ginzburg-Landau equation in the form used in nonlinear optics [10]: 


$$
\begin{gathered}
i \psi_{z}+\frac{1}{2} c \psi_{t t}+|\psi|^{2} \psi+\nu|\psi|^{4} \psi= \\
i \delta \psi+i \epsilon|\psi|^{2} \psi+i \beta \psi_{t t} \\
+i \mu|\psi|^{4} \psi
\end{gathered}
$$

where $z$ is the propagation distance, $t$ is the retarded time, $\psi$ is the normalized envelope of the field, $\delta$ is the linear gain coefficient, $i \beta \psi_{t t}$ accounts for spectral filtering, $\epsilon|\psi|^{2} \psi$ represents the nonlinear gain (which arises, e.g., from saturable absorption [11]), the term with $\mu$ represents, if negative, the saturation of the nonlinear gain, the one with $\nu$ corresponds to the saturation of the nonlinear refractive index, and $c= \pm 1$ depending on whether the group velocity dispersion is anomalous or normal, respectively. In this paper we shall consider the case $c=1$.

Several approaches have been used for the analysis of Eq. (1). Firstly, one can try to find stationary solutions of the CGL equation analytically (see, e.g., $[1,2,5,6])$. Unfortunately, only the cubic CGL equation can be completely analyzed in this way. Although the rich variety of stationary pulse solutions of the quintic CGL equation has been found [6], these solutions exist only in a subspace of the parameter space of a lower dimensionality. Moreover, these solutions (except the arbitraryamplicude pulses and flat-top pulses) are unstable.

Secondly, approximate methods such as perturbation theory (PT) can be applied [2,3]. In particular, the PT can be developed as an expansion around the one-soliton solution of the nonlinear Schrödinger equation. In this case, the PT predicts the existence of two motionless stationary solutions, one of them stable and the other unstable [3]. However, the PT can be applied only if the dissipative terms are small. All the solutions presented in this paper cannot be predicted by means of the PT.

Finally, one can solve Eq. (1) numerically, and this has been done in several papers $[3,7,8]$. However, such studies often give just fragmentary results, e.g., the existence of stationary pulses $[3,7]$ or the existence of bound states of two solitons [8]. The problem is that there are too many parameters involved, including the parameters of the initial conditions as well as the coefficients of the equation. More recent papers $[1,2]$ combine numerical simulations with an analytical study, which allows us to show an important relation between the front velocity and the stability of the pulses. In our recent paper [12] we have found numerically the range of existence of stable stationary pulses in the parameter space. This study is very important, because from whatever direction we move toward the region of the pulse existence and cross the stability boundary, we can expect some unusual dynamics.

This short comparison of different techniques shows that none of them is able to give an overall picture of what is happening with pulses. Only by combining them can one improve the understanding of the CGL equation and its solutions.

In our previous work [6] we concentrated our efforts on analytical considerations of the CGL equation. In this paper we look for qualitatively new solutions numerically.

\section{REDUCTION TO ODE SET}

One of the ways to find stationary solutions of Eq. (1) consists in its reduction to a set of ODE's. Namely, we seek the solution in the form

$$
\psi=a(\tau) \exp [i \phi(\tau)-i \omega z],
$$

where $a$ and $\phi$ are real functions of $\tau=t-v z, v$ is the pulse velocity and $\omega$ is the nonlinear shift of propagation constant. Substituting (2) into (1), we obtain an equation for two coupled functions: $a$ and $\phi$. Separating real and imaginary parts we have the following set of two ODE's:

$$
\begin{array}{r}
{\left[\omega-\frac{1}{2} c \phi^{2}+\beta \phi^{\prime \prime}+v \phi^{\prime}\right] a+2 \beta \phi^{\prime} a^{\prime}+\frac{1}{2} c a^{\prime \prime}+a^{3}+\nu a^{5}} \\
=0 \\
\left(-\delta+\beta \phi^{\prime 2}+\frac{1}{2} c \phi^{\prime \prime}\right) a+\left(c \phi^{\prime}-v\right) a^{\prime}-\beta a^{\prime \prime}-\epsilon a^{3}-\mu a^{5} \\
=0,
\end{array}
$$

where the prime denotes the derivative relative to the variable $\tau$. It can be transformed into

$$
\begin{aligned}
& \omega a+v \frac{M}{a}-\frac{1}{2} c M^{2} / a^{3}+\beta M^{\prime} / a+\frac{1}{2} c a^{\prime \prime}+a^{3}+\nu a^{5}=0, \\
& -\delta a-v a^{\prime}+\beta M^{2} / a^{3}+\frac{1}{2} c M^{\prime} / a-\beta a^{\prime \prime}-\epsilon a^{3}-\mu a^{5}=0,
\end{aligned}
$$

where $M=a^{2} \phi^{\prime}$. Separating derivatives we obtain:

$$
\begin{aligned}
M^{\prime}= & \frac{2(c \delta-2 \beta \omega)}{1+4 \beta^{2}} a^{2}+\frac{2(c \epsilon-2 \beta)}{1+4 \beta^{2}} a^{4} \\
& +\frac{2(c \mu-2 \beta \nu)}{1+4 \beta^{2}} a^{6}-\frac{4 \beta v}{1+4 \beta^{2}} M+\frac{2 c v}{1+4 \beta^{2}} a y \\
y^{\prime}= & \frac{M^{2}}{a^{3}}-\frac{2(c \omega+2 \beta \delta)}{1+4 \beta^{2}} a-\frac{2(c+2 \beta \epsilon)}{1+4 \beta^{2}} a^{3} \\
& -\frac{2(c \nu+2 \beta \mu)}{1+4 \beta^{2}} a^{5}-\frac{4 \beta v}{1+4 \beta^{2}} y-\frac{2 c v}{1+4 \beta^{2}} \frac{M}{a} \\
a^{\prime}=y &
\end{aligned}
$$

In principle, this set contains all stationary motionless and uniformly translating solutions. Parameters $v$ and $\omega$ are the eigenvalues of the set (5). On the plane $(M, a)$ the solutions corresponding to pulses are closed loops starting and ending at the origin.

If we are interested only in zero-velocity solutions $(v=$ $0)$, the set of Eqs. (5) becomes simpler:

$$
\begin{aligned}
M^{\prime}= & \frac{2(c \delta-2 \beta \omega)}{1+4 \beta^{2}} a^{2}+\frac{2(c \epsilon-2 \beta)}{1+4 \beta^{2}} a^{4} \\
& +\frac{2(c \mu-2 \beta \nu)}{1+4 \beta^{2}} a^{6}, \\
y^{\prime}= & \frac{M^{2}}{a^{3}}-\frac{2(c \omega+2 \beta \delta)}{1+4 \beta^{2}} a-\frac{2(c+2 \beta \epsilon)}{1+4 \beta^{2}} a^{3} \\
& -\frac{2(c \nu+2 \beta \mu)}{1+4 \beta^{2}} a^{5} \\
& a^{\prime}=y .
\end{aligned}
$$


Analytic one-soliton solutions can be found only at certain relations between the parameters [6]. However, this set of first order ODE's can be solved numerically. The asymptotic behavior of the set (6) at small $a$ is given by

$$
a=a_{0} \exp (g t)
$$

$$
M=\frac{c \delta-2 \beta \omega}{g\left(1+4 \beta^{2}\right)} a_{0}^{2} \exp (2 g t),
$$

where the soliton tail exponent $g$ can be found from the biquadratic equation

$$
g^{4}+\frac{2(c \omega+2 \beta \delta)}{1+4 \beta^{2}} g^{2}-\frac{(c \delta-2 \beta \omega)^{2}}{\left(1+4 \beta^{2}\right)^{2}}=0 .
$$

Namely,

$$
g^{2}= \pm \sqrt{\frac{\omega^{2}+\delta^{2}}{1+4 \beta^{2}}}-\frac{c \omega+2 \beta \delta}{1+4 \beta^{2}} .
$$

Using this approximation at the tails we can find the remaining part of any solution. The examples are given below.

\section{STATIONARY COMPLEX PULSES}

\section{A. Discovery of the stationary pulse}

Firstly, we recall results on the competition between pulses and fronts. To do this, we consider dynamics of a wide "rectangular" (e.g., super-Gaussian) pulse. If such a pulse is launched into the system, described by Eq. (1), first, the short transition to the coherent structure occurs. Such coherent structure is limited by two nonlinear fronts, i.e., interfaces between the cw and the background state. For the fixed coefficients of the equation, the family of nonlinear fronts exists. However, only fronts with some particular values of the amplitude, wave vector, and velocity can be formed. This process is governed by the front selection criteria. This means that front and source should match together on two parameters: amplitude and wave vector. So, some particular value of the front velocity is selected. Other possibilities also exist such as fronts themselves may be unstable, but we do not consider them here.

This selected value of velocity determines the further dynamics of the coherent structure. We define the front velocity as negative if the background state spreads over the $\mathrm{cw}$. Otherwise, the front velocity is positive. So, if the velocity is negative, the structure shrinks. Normally, when two fronts become close to each other, they form a stationary pulse [Fig. 1(a)]. However, one more possibility exists and it is shown in Fig. 1(b). Finally, if the velocity is positive, the structure expands [Fig. 1(c)].

As one can see, the new form of localized solution is formed, which we call composite pulse. Apparently, it exists if the front velocity has small negative values. To find the range of parameters, where the CP exists, its properties, and the possibility of coexistence with plain pulse, we performed a series of computations, described below.
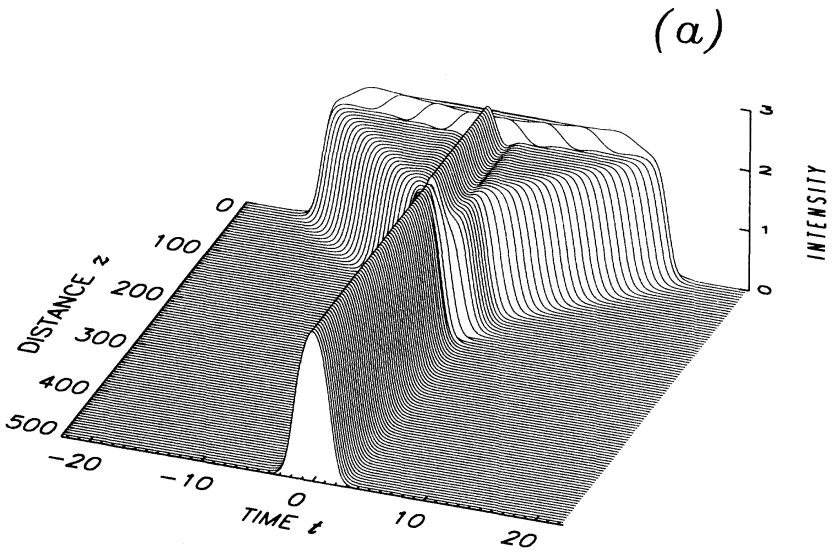

(b)
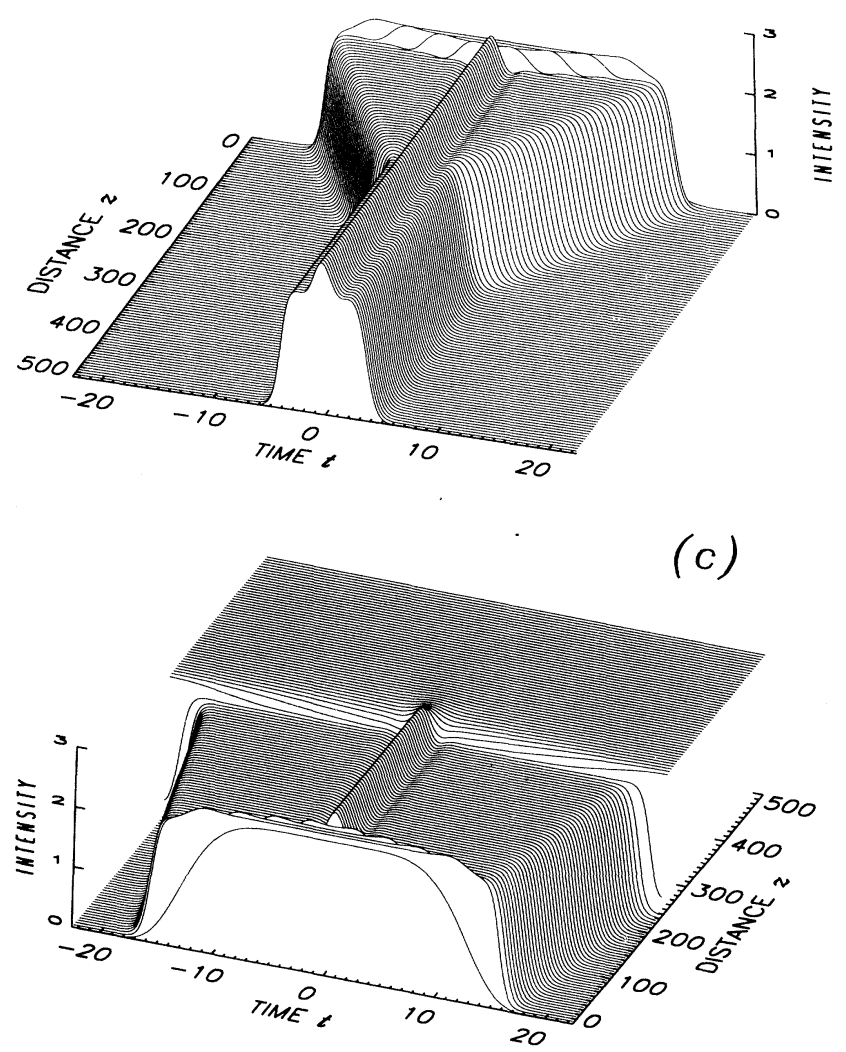

FIG. 1. Dynamics of the coherent structure consisting of two fronts and source, $\delta=-0.5, \beta=0.5, \mu=-1, \nu=-0.1$. (a) $\epsilon=2.51$, the structure shrinks and the plain pulse is formed; (b) $\epsilon=2.52$, the structure shrinks and the composite pulse is formed; (c) $\epsilon=2.53$, the structure expands. Note that the position of the viewpoint in (c) is different from (a) and (b). 


\section{B. Range of existence of the CP}

First of all, we looked for the range of parameters where the plain pulse exists. For this task, we solved numerically Eq. (1) to obtain the stationary pulse solution, and then slightly varied one of the coefficients (normally $\epsilon)$ to find the stationary pulse for different parameters. Note that we followed two different approaches. In the first approach, the same initial condition (say, sech type or Gaussian function) is used after each change of the parameters of the equation. It is usually supposed that for the quintic CGL equation there is only one localized stable stationary solution for each set of parameters, so if the initial condition evolves to some stationary state, it is supposed to be this solution. When using the other method, after each small modification of the parameters the stationary solution found in the previous step is used as an initial condition for the next step. This method provides much faster convergence. If there is only one stable or quasistable nonzero solution, then there is no difference between these two approaches. However, if there are two (or more) stable solutions for the same set of parameters, then the choice of the initial condition becomes crucial.

To choose the set of parameters, we note that the stable plain pulses exist only when $\delta<0, \beta>0, \epsilon>0$, and $\mu<0$. The parameter $\nu$ can be both positive or negative. In fact, these conditions mean that one term (that with coefficient $\epsilon$ ) provides gain to the pulse and three other terms (those with coefficients $\delta, \beta$, and $\mu$ ) produce losses.

We choose $\delta=-0.1, \beta=0.5, \nu=-0.1$ in all simulations presented in this paper. Next, we varied the parameters $\epsilon$ and $\mu$ to find roughly the threshold of positive front velocity. The initial point found was $\mu=-0.6$, $\epsilon=1.8$. Further simulations have been carried out starting from the vicinity of this point.

To find the threshold of the positive front velocity more precisely, we choose the initial point on the $(\mu, \epsilon)$ plane at the point $(-0.6,1.6)$, obtained the stationary plain pulse, and then employed the second method, i.e., increased $\epsilon$ slightly to obtain a new stable solution taking as initial condition the previously found stable solution for lower $\epsilon$, and repeated the procedure. In this way we can obtain the continuous dependence of the pulse energy and any other pulse characteristics on the parameter $\epsilon$ (see Fig. 2). A more precise value of the positive front velocity threshold has been found to be $\epsilon=1.78$.

Next, keeping the same set of parameters, we applied the first method. Namely, the same initial condition $\left[\psi_{0}(t)=\operatorname{sech}(t)\right]$ has been used after each variation of $\epsilon$. In this case the results coincide with those obtained using another method up to $\epsilon=1.7$. Above this value there are some oscillations around the previous results, and at $\epsilon=1.75$ we observed the convergence to a new type of solution, namely, the composite pulse. We choose this term because this solution is considered to be composed from a source and two fronts, as discussed below.

In the next series of computations we started from $\psi_{0}(t)=\operatorname{sech}(t)$ at $\epsilon=1.75$, obtained the composite pulse, and then varied $\epsilon$, again using the stable solution

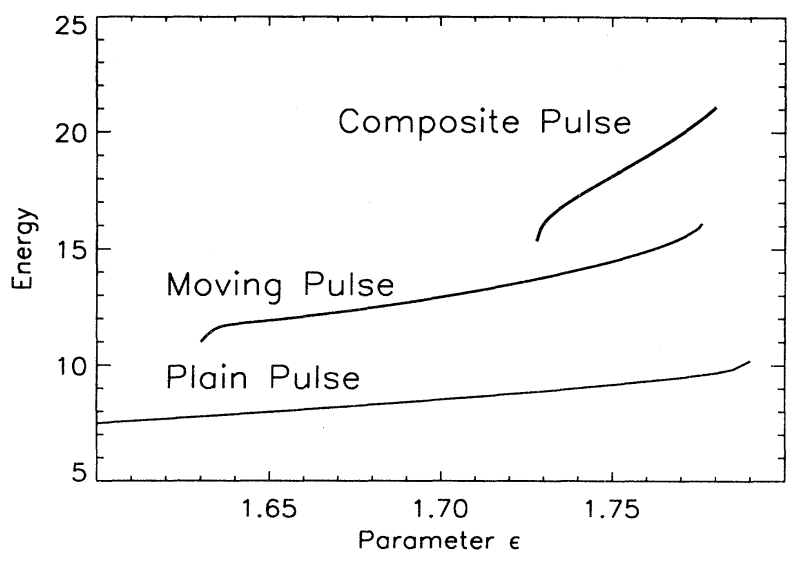

FIG. 2. The energies of the plain pulse, the composite pulse, and the moving pulse vs $\epsilon, \delta=-0.1, \beta=0.5, \mu=-0.6$, $\nu=-0.1$.

found in the previous step as the initial conditions. In this way we were able to produce the curve that shows the CP energy (see Fig. 2). As we increased $\epsilon$, we realized that the range of existence of the $\mathrm{CP}$ is also limited by the positive front velocity threshold.

This procedure has been repeated for different $\mu$. The results are summarized in Fig. 3. It shows the ranges of parameters where the stable plain pulses and the composite pulses exist. The upper boundary on the $(\mu, \epsilon)$ plane corresponds to the transition into pair of fronts. Dynamics of the CP at the lower boundary is discussed below.

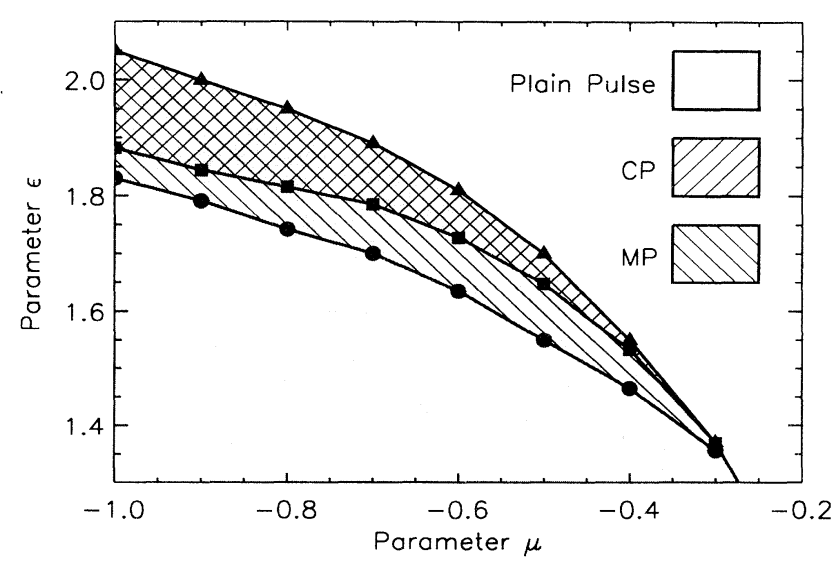

FIG. 3. The range of existence of the stationary stable pulses on the $(\mu, \epsilon)$ plane. The solid line marked by triangles gives the zero front velocity threshold, and filled areas give the ranges of existence of the plain pulse, the composite pulse, and the moving pulse. Parameters are $\delta=-0.1$, $\beta=0.5, \nu=-0.1$. 


\section{Properties of the composite pulse}

The profile, of a CP, its phase profile and Fourier transform are shown in Fig. 4. The same curves for the plain pulse solution that exists for the same set of parameters are also given for comparison. The CP consists of two fronts and a small "hill" between them. This hill is the domain boundary between two fronts, as they have nonzero wave vectors. This hill should be counted as a source, because it follows from the phase profile that there is an energy flow from the center to the CP wings. Note that the flat regions between the source and the fronts are relatively small. The typical width of the source is the same as the typical width of the front.

To compare the CP with the plain pulse we use the presentation on the $(a, M)$ plane (see Sec. III). From Fig. 5 we realize that that there are many similarities between the two solutions. In particular, the top part of the CP (i.e., the source) has the same shape as the top part of the pulse. The wings of the simple pulse and the CP are also very similar. To explain this fact, we compared the nonlinear propagation constants of both structures. It appears that the difference is around $10 \%$. Then the similarity between the asymptotes follows from the linearized version of the Eq. (1).

The spectrum of the CP is shown in Fig. 4(c). It has a hole in its center and two well-separated peaks. To explain such structure of the spectrum, let us return to the dynamical coherent structure, considered above. Let us suppose that the full width of the structure is much larger than the width of both the front and the source. So, it consists mainly of two plane waves located between the source and two fronts. These plane waves have opposite
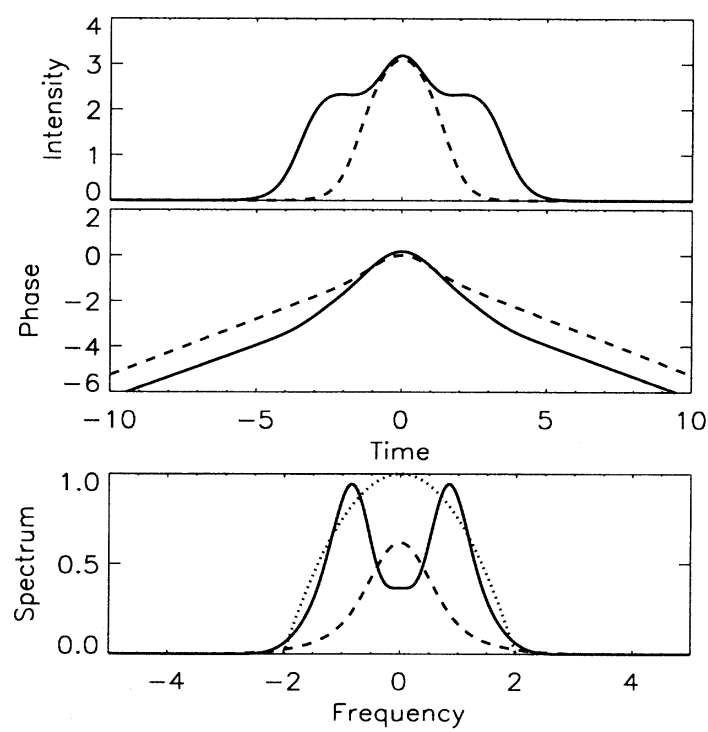

FIG. 4. The amplitude, phase profiles, and the spectrum of the composite pulse (solid curves) and plain pulse (dashed curves), $\delta=-0.1, \beta=0.5, \epsilon=1.75, \mu=-0.6, \nu=-0.1$. Dotted curves give the width of the spectral filtering $1-\beta^{2} \omega^{2}$.

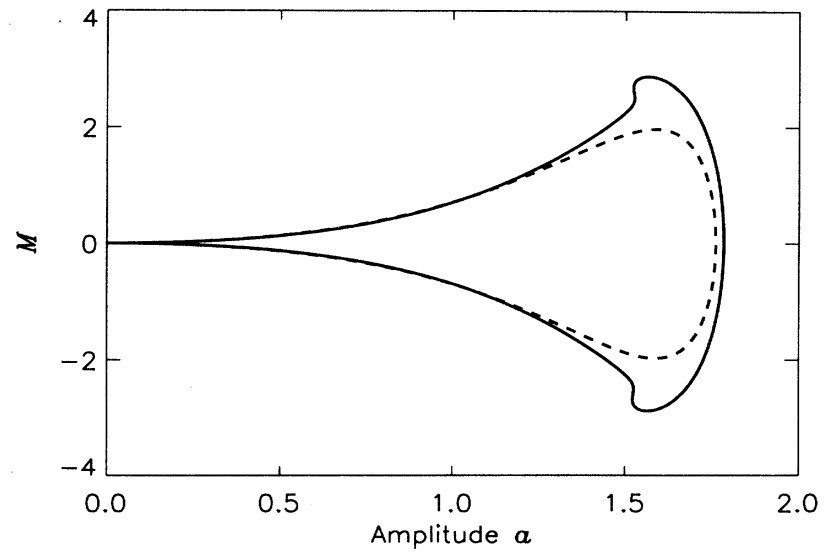

FIG. 5. Comparison of the plain pulse (dashed curve) and the CP on the $(a, M)$ plane. The parameters are as in Fig. 4.

wave vectors, and the spectrum of this structure consists of two peaks. The spectral separation between the peaks is determined by the difference between the wave vectors of the two plane waves, and it roughly does not change if the whole structure propagates (and expands or shrinks). But the width of each peak varies. For instance, if the structure expands, the width of each peak decreases and vice versa. The stationary $\mathrm{CP}$ is the limiting case of the dynamical case, and its spectrum is the intermediate case between the spectrum of the dynamical structure, which has two peaks, and the spectrum of the plain pulse, which has one peak.

\section{Discussion}

As far as we know, these results are the first demonstration of the coexistence of two different stable stationary solutions of the quintic CGL for the same set of parameters. This fact contradicts arguments derived from the perturbation around the conservative and purely dissipative limit. At the same time, this result is quite natural, if we consider fronts, pulses, and sources as elementary building blocks, which can be combined to form more complicated structures.

The CP solutions exist in the range of parameters where the selected front has small negative velocity. So, we can explain the existence of the CP by some repulsion that exists between the source and the front. When the front velocity is small, as structure shrinks and fronts move toward each other, they meet this repulsion from the source, which keeps them at a fixed distance. For the other set of parameters, where the front velocity is large enough, they overcome this repulsion and the plain pulse is formed. However, this qualitative approach does not explain why the CP can be formed from an initial condition that is much closer to the plain pulse than to the CP. Nevertheless, some additional arguments that support the idea of repulsion between the front and the source will be given below. 
Note also that there is some range of parameters where the selected front has zero wave vector. Apparently, in this case the plain pulse and the CP become indistinguishable.

\section{E. Dynamics of the composite pulses}

Now, let us turn back to Fig. 3 and consider more carefully the range of existence of composite pulses. The upper boundary on this plot corresponds to the positive front velocity threshold. The lower boundary corresponds, in general, to the transition from the composite pulse to the plain pulse. However, this transition is not straightforward.

In particular, we have discovered the existence of periodic solutions in this range of parameters. In Fig. 6 we show the dynamics of the pulse energy versus $z$ for two values of $\epsilon$ near the lower boundary. As one can see, pulses demonstrate complicated periodical dynamics, with main period varying from $T=69$ (for $\epsilon=1.726$ ) to $T=95$ (for $\epsilon=1.727$ ). Note that when the pulse energy has the lowest value at each period, this value is much smaller than the energy of the plain pulse. Hence, this dynamics cannot be considered as a periodical transition between these two types of solutions.

Our calculations show that such periodic dynamics can be observed in a very narrow interval of parameters and only for some particular initial condition. If we slightly increase $\epsilon$, stationary CP is formed. If $\epsilon$ is decreased, the solution is transformed into the plain pulse or moving pulse, described below.

Note that periodical dynamics of pulses for the quintic GL equation has been reported recently in Ref. [13]. However, there are important differences between our results and the results of Ref. [13]. We observed instability of the composite pulse, while the authors of Ref. [13] report the instability of plain pulses. Then, in our case

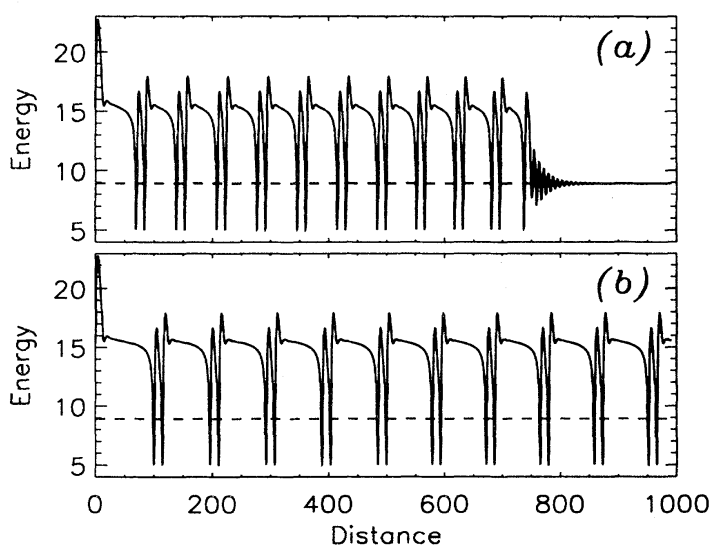

FIG. 6. Periodical dynamics of the complex pulse at (a) $\epsilon=1.726$ and (b) $\epsilon=1.727$. Dashed line gives the energy of the plain pulse. Other parameters are $\delta=-0.1, \beta=0.5$, $\mu=-0.6, \nu=-0.1$. the changes are deeper, and the pulse energy varies more than 3 times. Dynamics, reported in Ref. [13], is caused by the instability of fronts, while the pulse in general remains stable. Consequently, the pulse energy does not vary more than $20 \%$. Finally, if we detune the parameters slightly, periodical dynamics disappears, and a stationary solution is formed, while in Ref. [13] periodical dynamics becomes quasiperiodic or chaotic.

\section{MOVING PULSES}

\section{A. Discovery and properties of moving pulses}

Traditionally, it is supposed that if the coefficient $\beta$ in front of the second-order derivative term in the righthand side (rhs) of Eq. (1) is nonzero, then only motionless and symmetric localized stationary solutions can exist [14]. This follows, for example, from the adiabatic perturbation theory (see, e.g., [9] and references therein). However, perturbation theory cannot be applied if at least one of the coefficients in the rhs of Eq. (1) is not small. It has been proven already that asymmetric solutions of the symmetric nonlinear problem can exist in the conservative case [15], so we can expect this to be true for the non-conservative problem as well.

MP can be observed as a result of the instability of the $\mathrm{CP}$ at the lower boundary of the region of stable $\mathrm{CP}$ on the $(\mu, \epsilon)$ plane. If the antisymmetric perturbation has a large growth rate, then instead of the periodic dynamics described above or transformation into the plain pulse, we would have the spontaneous transformation into asymmetric MP (Fig. 7). The process of CP formation can be controlled by choosing a moving initial condition. In this case both left- and right-moving pulses can be created; they are related as an original and a mirror image, due to the symmetry of Eq. (1) relative to the transformation $t \leftrightarrow-t$.

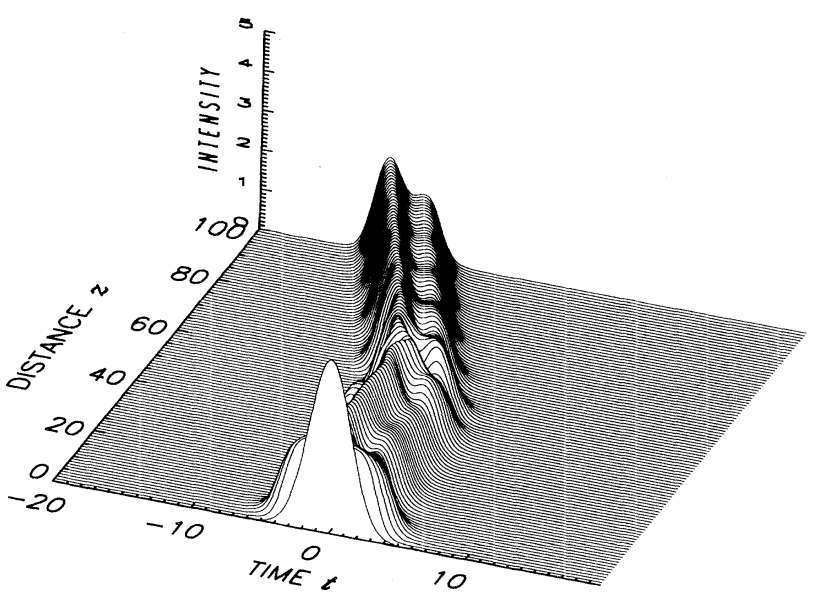

FIG. 7. Spontaneous transformation of the composite pulse into the moving pulse, $\delta=-0.1, \beta=0.5, \nu=-0.1, \epsilon=1.8$, $\mu=-0.8$. 
The amplitude, phase profile, and the spectrum of the MP are given in Fig. 8. The amplitude profile is indeed very close to the profiles of the plain pulse and the composite pulse. In other words, MP can be considered as the bound state (nonlinear superposition) of the plain pulse and front, or as a CP in which one of the fronts is missing. The spectrum of the moving pulse is asymmetric. There are two peaks of different height and a hole between them.

It follows from the presentation of the MP as the bound state of a pulse and a front that its energy can be roughly presented in the form $E_{\mathrm{MP}}=E_{P}+E_{F}$, while the energy of the composite pulse takes the form $E_{\mathrm{CP}}=E_{P}+2 E_{F}$, where $E_{P}$ is the energy of the plain pulse. In other words, the difference between the MP energy and the energy of the plain pulse (which exists for the same set of parameters) is half of the difference between the energy of the CP and plain pulse. Numerical calculations show qualitative agreement with this prediction (see Fig. 2).

It is noticeable that MP always moves with the pulse ahead. This confirms our hypothesis that the MP is the nonlinear combination of a pulse and a front, because it exists in the region of parameters where the front velocity is negative. Another important fact is that the velocity of MP is always smaller than the velocity of the selected front for the same set of parameters. To explain this, we note that, in the MP, the front tends to move with its own velocity, i.e., the velocity of the selected front. At the same time, the pulse tends to be stationary due to the spectral filtering. The resulting velocity of the MP is determined by the competition between these two processes. At the same time, the distance between the center of the pulses and the front in MP is slightly larger
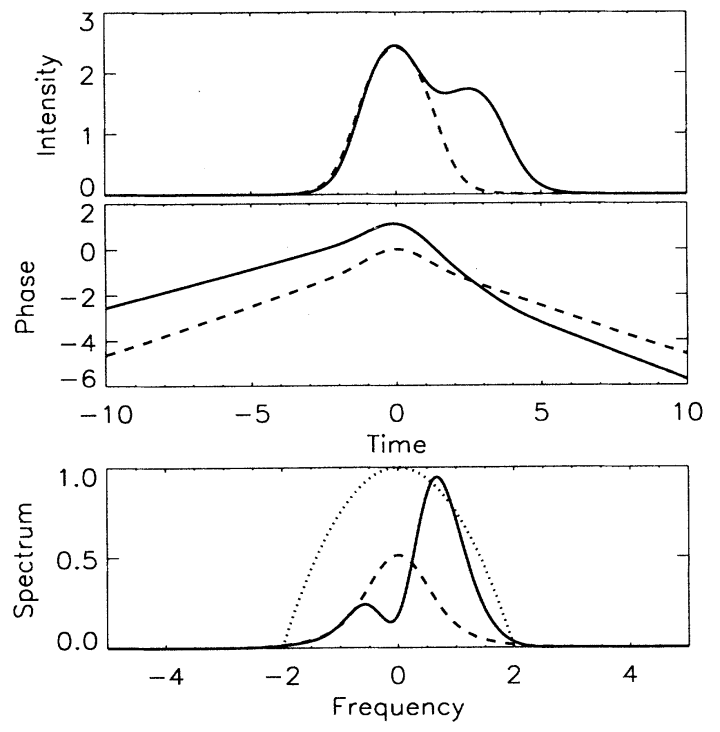

FIG. 8. The amplitude, phase profiles, and the spectrum of the moving pulse (solid curves) and simple pulse (dashed curves), the parameters are the same as in Fig. 7. Dotted curves give the width of the spectral filtering $1-\beta^{2} \omega^{2}$. than the same distance in the CP, because the pulse offers less resistance to the pushing force of the front. This explains the difference between the predicted and the observed energy of the MP.

Note the difference between the MP observed in our paper and asymmetric stationary solution presented in [15]. In our case, the medium is homogeneous, while in [15] there is symmetric inhomogeneity. At the same time, the presence of spectral filtering can be considered as inhomogeneity in the frequency domain. In both cases, the asymmetric pulse arises near the boundary, in the space domain in [15] and in the frequency domain in our case.

To find the range of existence of the MP, we started from the moving initial condition in the range of parameters where the CP exist, and then slowly decreased $\epsilon$. It turns out that the range of existence of the MP is even larger than for the CP (see Fig. 2). The upper boundary is the same for plain pulses, the CP and the MP, while the range of existence of the MP is approximately 2 times wider. However, both the CP and the MP do not exist for $\mu>-0.3$. Apparently, this is the threshold where the double matching (between amplitudes and wave vectors) of the pulse and front becomes possible. As we decrease $\mu$ below -0.3 , the range of this matching increases. This again proves our hypothesis that the CP and the MP have common nature.

Note also that the MP exists in the continuous range of parameters and this range is comparable with the range of existence of the plain pulses. Apparently, the MP have been missed in previous analyses because it has been supposed that only motionless pulses can exist and corresponding initial conditions have been used.

At the lower boundary of their existence on the $(\mu, \epsilon)$ plane, the MP are transformed into a plain pulses. This process can be explained in the following way: As the front velocity increases, resistance from the pulse also increases. At some point the front velocity overcomes the repulsion and the front is absorbed by the pulse. In this sense the lower boundaries for the CP and the MP on the $(\mu, \epsilon)$ plane have the same meaning.

To avoid any misunderstanding, we have to explain the difference between our moving pulses and moving pulses in Refs. $[8,16,17]$. Our moving pulses are moving due to some internal reasons. By contrast, pulses in $[8,16,17]$ were moving due to external factors, i.e., group velocity terms and gradient terms. Neither group velocity nor gradient terms were present in our simulations. Also, the MP in our simulations has asymmetry as an intrinsic feature, while pulses in Ref. [16] become asymmetric under the action of gradient terms.

\section{B. Interaction of moving and stationary pulses}

There are four stable pulse solutions in some regions of the parameter space, namely, plain pulse, composite pulse, and moving to the right and to the left pulses. This gives three possibilities of pair interaction, i.e., interaction of moving pulse with the plain pulse, with the 
composite pulse, and with another moving pulse. Note that the result of the collision depends also on the relative phase of the interacting pulses. For a collision between moving and motionless pulses that have different propagation constants, the relative phase at the collision point depends on both the initial phase difference and the initial separation, so it is difficult to control the relative phase in numerical simulations. If two moving pulses collide, the relative phase between them is determined
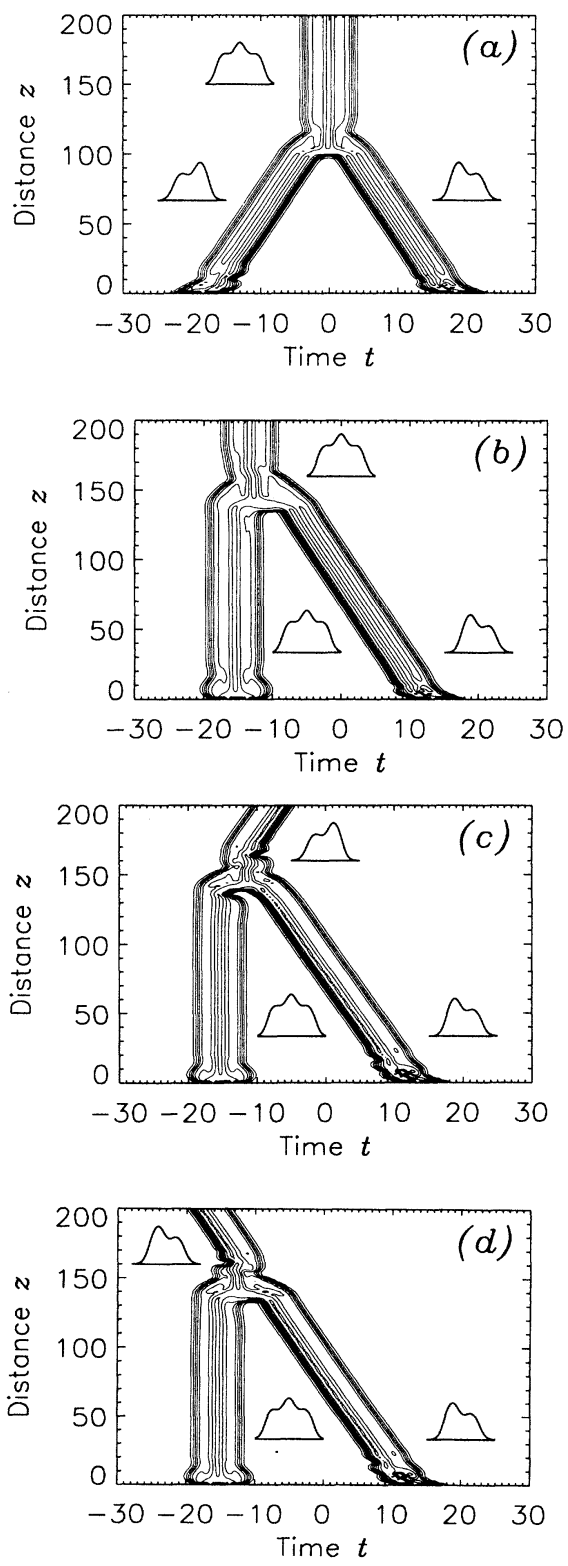

FIG. 9. Interaction between the moving and composite pulses, $\delta=-0.1, \beta=0.5, \nu=-0.1, \mu=-0.6$. (a) Interaction between the in-phase MP, $\epsilon=1.75$; (b) interaction between the MP and the CP, $\epsilon=1.75$; (c) interaction between the MP and the CP, $\epsilon=1.73$ (note that after the collision the orientation of the MP is opposite to the initial one); (d) interaction between the MP and the CP, $\epsilon=1.73$, the relative phase is changed on $\pi$ in comparison with (c). only by the initial phase difference, so it can be easily controlled.

Figure 9 depicts different scenarios of interaction. We start from the interaction between the MP. In this case we observed either the formation of the CP [for in-phase MP, see Fig. 9(a)], or the plain pulse, or another MP (for out-of-phase pulses). Collisions between stationary CP and MP also result in several possibilities. Figure $9(\mathrm{~b})$ shows one of them, namely, absorption of the MP by the CP. The only difference between the CP before and after the collision is the small shift on $t$.

Figures 9(a) and 9(b) have been obtained for $\epsilon=1.75$. As we decrease $\epsilon$ to 1.73 , the CP still exists, but its stability wanes. For this set of parameters we observed formation of the MP after the collision, and direction of propagation of this MP depends on the phase difference between the pulses [Figs. 9(c) and 9(d)]. Note that if the MP changes its direction of motion after collision, as in Fig. 7(c), it also changes its orientation, because MP always moves with the pulse ahead, front behind. Potentially, there are other possible results of interaction, including complete annihilation of interacting pulses and tunneling of one pulse through another one. We expect to observe these scenarios in future simulations.

We note the difference between our results and the results of Refs. $[8,16,17]$. In these papers, interaction in the frame of two coupled GL equations has been studied, which described propagation of two components. Also, as we already mentioned, the pulses were uniformly translating due to group velocity terms, incorporated into the equations. So, in each component, only one direction of motion is possible. By contrast, in our case we consider one GL equation, the pulses move due to internal reasons, and both left- and right-moving pulses are possible. At the same time, there are a number of similarities between our results and the results of $[8,16,17]$. In particular, the disappearance of one of the pulses after the interaction has been found in [17]. The fusion of two pulses into one has been observed in [16], although for the case where the resulting two-component pulse was unstable and it transformed into two fronts.

\section{DISCUSSION AND CONCLUSION}

In this paper we have found numerically a series of new solutions of the CGL equation. To explain their existence, we propose the hypothesis that pulses, fronts, and sources can be considered as elementary building blocks, which can be combined to form more complicated structures. Another example of a stationary solution, which arises from the superposition of two simplest solutions, is the bound state of two pulses $[2,8,18]$. However, pulses in such bound state are weakly overlapping. Moreover, as we demonstrated recently, the bound states of two pulses are unstable [18]. At the same time, the bound states of two pulses exist in a relatively wide range of parameters. By contrast, we report stable structures, which arise as a result of strong interaction between the pulses and fronts. However, such structures require the matching conditions for the amplitude and the wave vector to be fulfilled, so 
they can exist in a relatively narrow range of parameters.

In conclusion, we study numerically propagation of localized coherent structures in the quintic GinzburgLandau equation near the zero front velocity threshold. We have found that besides the stationary pulse solution, known before, another type of stationary solution, which we call the composite pulse, can exist. The composite pulse and the plain pulse exist at the same values of parameters. Also, we show that such composite pulses can demonstrate periodic dynamics. We discovered the existence of the asymmetric moving pulses and studied the interaction between the moving the motionless pulses.
To explain the existence of the structures observed, we suppose that the simple pulse and nonlinear front can be considered as elementary building blocks, which can form more complicated nonlinear superpositions.

\section{ACKNOWLEDGMENTS}

The work of V.V.A. and N.A. was supported by the Australian Photonics Cooperative Research Centre (APCRC). The work of J.M.S.-C. was supported by the CICyT under Contract No. TIC95-0563-03.
[1] W. van Saarloos and P.C. Hohenberg, Phys. Rev. Lett. 64, 749 (1990).

[2] W. van Saarloos and P.C. Hohenberg, Physica (Amsterdam) 56D, 303 (1992).

[3] S. Fauve and O. Thual, Phys. Rev. Lett. 64, 282 (1990).

[4] V. Hakim, P. Jakobsen, and Y. Pomeau, Europhys. Lett. 11, 19 (1990).

[5] P. Marcq, H. Chatê, and R. Conte, Physica (Amsterdam) 73D, 305 (1994)

[6] N. Akhmediev and V.V. Afanasjev, Phys. Rev. Lett. 75, 2320 (1995); N.N. Akhmediev, V.V. Afanasjev, and J.M. Soto-Crespo, Phys. Rev. A (to be published).

[7] O. Thual and S. Fauve, J. Phys. (Paris) 49, 1829 (1988).

[8] H.R. Brand and R.J. Deissler, Phys. Rev. Lett. 63, 2801 (1989).

[9] C. Elphick and E. Meron, Phys. Rev. Lett. 65, 2476 (1990).

[10] J. D. Moores, Opt. Commun. 96, 65 (1993).

[11] V.V. Afanasjev, Opt. Lett. 20, 704 (1995).
[12] J.M. Soto-Crespo, N. Akhmediev, and V. Afanasjev (unpublished).

[13] R.J. Deissler and H.R. Brand, Phys. Rev. Lett. 72, 478 (1994).

[14] Strictly speaking, the existence of the moving pulses has been mentioned once in the literature. We cite from Ref. [1]: for the fixed coefficients "one also finds a discrete set of moving pulse solutions with specified velocity $v_{p}$ and frequency $\omega_{p}$, plus a symmetric stationary pulse $\left(v_{\mathrm{sp}}=0, \omega_{\mathrm{sp}}\right)$, which exists for generic parameter values."

[15] N.N. Akhmediev, Zh. Eksp. Teor. Fiz. 83, 545 (1982) [Sov. Phys. JETP 56, 299 (1982)].

[16] R.J. Deissler and H.R. Brand, Phys. Lett. A 146, 252 (1990).

[17] R.J. Deissler and H.R. Brand, Phys. Rev. Lett. 74, 4847 (1995).

[18] V.V. Afanasjev and N. Akhmediev, Opt. Lett. 20, 1970 (1995). 


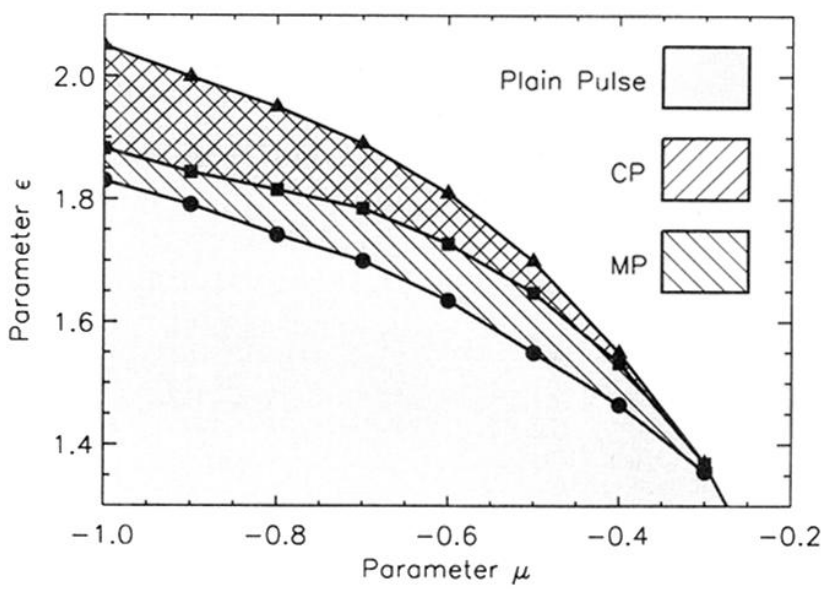

FIG. 3. The range of existence of the stationary stable pulses on the $(\mu, \epsilon)$ plane. The solid line marked by triangles gives the zero front velocity threshold, and filled areas give the ranges of existence of the plain pulse, the composite pulse, and the moving pulse. Parameters are $\delta=-0.1$, $\beta=0.5, \nu=-0.1$. 